\title{
Pion-pion scattering lengths from Ke4 and Cusp at NA48/2 experiment
}

\author{
G. Lamanna ${ }^{\mathrm{a} *}$ \\ ${ }^{a}$ Dipartimento di Fisica \& INFN PISA, \\ Largo Pontecorvo,3 50127 Pisa, Italy
}

In kaon decays the measurement of $\pi \pi$ scattering lengths ( $a_{0}$ and $\left.a_{2}\right)$ are accessible mainly through the study of kinematical properties in two decay modes. The classic way is based on the form factors analysis in $K^{ \pm} \rightarrow$ $\pi^{+} \pi^{-} e^{ \pm} \nu\left(K_{e 4}\right)$ decay. Thanks to the absence of other hadrons in the final state the $\pi \pi$ interaction can be analyzed in terms of phase shift variation with the $\pi \pi$ invariant mass. The preliminary results on a set of $\sim 1150000 K^{ \pm}$ events collected in two years of data taking, will be discussed. The second way to evaluate the $\pi \pi$ scattering lengths is based on the accurate study of $K^{ \pm} \rightarrow \pi^{ \pm} \pi^{0} \pi^{0}$ Dalitz plot, in which the strong rescattering process $\pi^{+} \pi^{-} \rightarrow \pi^{0} \pi^{0}$ introduces a singularity in the shape of the distribution. The preliminary result based on more than $\sim 60 \cdot 10^{6} K^{ \pm} \rightarrow \pi^{ \pm} \pi^{0} \pi^{0}$ decays is in good agreement with the measurement in $K_{e 4}$.

\section{EXPERIMENTAL SETUP}

The NA48/2 experiment beam line has been designed to measure the $\mathrm{CP}$ violating charged asymmetry in the $K \rightarrow 3 \pi$ decay [1]. Simultaneous positive and negative kaons beams are produced in the same beryllium target by impinging $400 \mathrm{GeV}$ protons from the CERN/SPS accelerator. The momentum range of $(60 \pm 3) \mathrm{GeV} / \mathrm{c}$ is selected, for both beams, in the first "achromat" and a complex system of magnets allows to have superimposed and focused beams $\sim 200 \mathrm{~m}$ downstream, at the end of the $\sim 100 \mathrm{~m}$ long decay region. A schematic view of the beam line can be found in [1]. Both $K^{+}$and $K^{-}$decays are collected in the same NA48 detectors, described elsewhere [2]. The $K_{e 4}$ analysis is essentially based on the magnetic spectrometer, consisting of a magnet dipole and two sets of two drift chambers with a momentum resolution of $\sigma(p) / p=(1.0 \oplus 0.044 \cdot p) \%$ (with $\mathrm{p}$ in $\mathrm{GeV} / \mathrm{c}$ ). The $K^{ \pm} \rightarrow \pi^{ \pm} \pi^{0} \pi^{0}$ analysis uses the electromagnetic calorimeter to identify the gammas produced in $\pi^{0}$ decay. The E.M. calorimeter has $\sim 27$ radiation lengths of liquid krypton (LKr) with a resolution of $\sigma(E) / E=(3.2 / \sqrt{E} \oplus 9.0 / E \oplus 0.42) \%$ (with $\mathrm{E}$ in $\mathrm{GeV}$ ).

*Present address: Scuola Normale Superiore, Piazza dei Cavalieri,7 50126 Pisa, Italy

\section{THE $K_{e 4}$ DECAY}

The $K_{e 4}$ kinematics is fully described using the 5 Cabibbo-Maksymowicz variables [3]. Thanks to these variables the two axials $(F, G)$ and one vectorial $(\mathrm{H})$ form factors that contribute to the transition amplitude can be written in terms of partial wave expansion [4]:

$$
\begin{aligned}
& F=F_{s} e^{i \delta_{s}}+F_{p} e^{i \delta_{p}}+\ldots \\
& G=G_{p} e^{i \delta_{p}}+\ldots \\
& H=H_{p} e^{i \delta_{p}}+\ldots
\end{aligned}
$$

the four form factors can be further expanded in powers of $q^{2}=\left(M_{\pi \pi}^{2} / 4 m_{\pi}^{2}\right)-1$ :

$$
\begin{aligned}
& F_{s}=\left(f_{s}+f_{s}^{\prime} q^{2}+f_{s}^{\prime \prime} q^{4}\right) \\
& F_{p}=f_{p} \\
& G_{p}=\left(g_{p}+g_{p}^{\prime} q^{2}\right) \\
& H_{p}=h_{p}
\end{aligned}
$$

(where $M_{\pi \pi}$ is the pions pair invariant mass) and only the phase shift $\delta\left(q^{2}\right)=\delta_{s}-\delta_{p}$ is taken into account. About 1150000 decays were selected looking for events in which three good reconstructed tracks are identified in the magnetic spectrometer. The particle identification exploits the ratio between the energy measured in the calorimeter and the momentum in spectrometer. The background is evaluated by studying the "wrong" sign events, i.e. the events for which the $\Delta S=\Delta Q$ rule is violated (we are not 
Table 1

Form factors. Preliminary results on $2003+2004$ data.

\begin{tabular}{|c|c|c|c|c|c|}
\hline$f_{s}^{\prime} / f_{s}$ & $=$ & $0.158 \pm 0.007 \pm 0.006$ & $g_{p} / f_{s}$ & $=$ & $0.869 \pm 0.010 \pm 0.012$ \\
\hline$f_{s}^{\prime \prime} / f_{s}$ & $=$ & $-0.078 \pm 0.007 \pm 0.007$ & $g_{p}^{\prime} / f_{s}$ & $=$ & $0.017 \pm 0.022 \pm 0.015$ \\
\hline$f_{e}^{\prime} / f_{s}$ & $=$ & $0.067 \pm 0.006 \pm 0.009$ & $h_{p} / f_{s}$ & $=$ & $-0.402 \pm 0.014 \pm 0.008$ \\
\hline$f_{p} / f_{s}$ & $=$ & $-0.049 \pm 0.003 \pm 0.004$ & & & \\
\hline
\end{tabular}

Both statistical and systematical errors are shown.

For this preliminary result the systematical error is conservatively quoted from the 2003 data only.

Table 2

Scattering lengths results

\begin{tabular}{lll}
\hline & \multicolumn{1}{c}{2003 data (published) } & $2003+04$ data (preliminary) \\
\hline a0 free & $0.209 \pm 0.016 \pm 0.007$ & $0.218 \pm 0.013 \pm 0.007$ \\
$\mathrm{a} 2$ free & $-0.0529 \pm 0.0105 \pm 0.0041$ & $-0.0457 \pm 0.0084 \pm 0.0041$ \\
\hline $\mathrm{a} 0$ (ChPT) & $0.223 \pm 0.006 \pm 0.002$ & $0.220 \pm 0.005 \pm 0.002$ \\
\hline
\end{tabular}

The systematical error in the $2003+2004$ preliminary results is conservatively

quoted from the 2003 data.

sensitive to measure this violation) and it is at level of $0.5 \%$. In order to fit the form factors and the phase shift, the whole data sample has been subdivided in 15000 bins defined in the CabibboMaksymowicz variables 5 dimensional space, and a GEANT3 [5] based montecarlo has been employed to describe the detector acceptance. In particular in each of the 10 bins along $M_{\pi \pi}$ a 4 parameters fit was performed in order to extract the form factors. In tab. 1 the form factors results, based on the data collected during the 2003 and 2004 run, are summarized. For this preliminary result, the systematics are conservatively quoted from 2003 data [6].

A second fit is made to determine the form factors $q^{2}$ dependence. In the case of the phase shift $\delta$, the Roy equations [7] are used to fit the $q^{2}$ dependence. Through the Roy equations, after an extrapolation from $\pi \pi$ scattering data at higher energy, it is possible to correlate the $\delta$ phase shift to the scattering lengths $a_{0}$ and $a_{2}$. In fig. 1 the fitting result is shown. According to [8] the isospin correction in each point can't be ignored being of the same order of magnitude with respect to the statistical error $(\sim 13 \mathrm{mrad}$ depending on the point). Exploiting a $a_{2}=f\left(a_{0}\right)$ relation given, for instance, by the ChPT [9], it's possible to perform a 1 parameter fit in the uni-

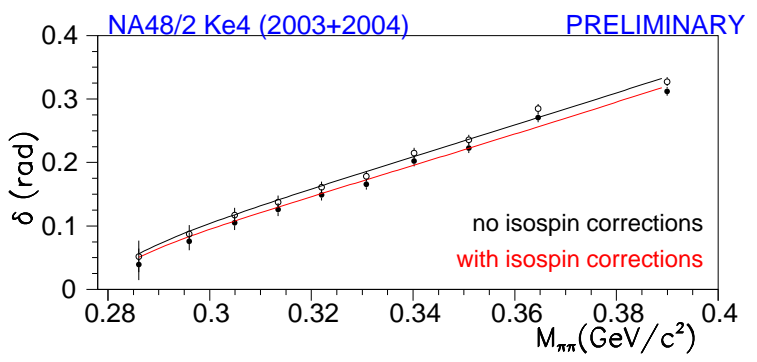

Figure 1. Phase shift as a function of $M_{\pi \pi}$

versal band (the universal band is the region in the $a_{0}$ and $a_{2}$ plane defined by the extrapolation from high energy experimental data to the threshold region). The table 2 shows the results using only the Roy Equations and the ChPT relation, with the comparison between the preliminary results presented here and the already published results [6] based on a partial data set.

\section{THE CUSP ANALYSIS}

The pion scattering lengths can also be measured through the study of the "cusp" in the 


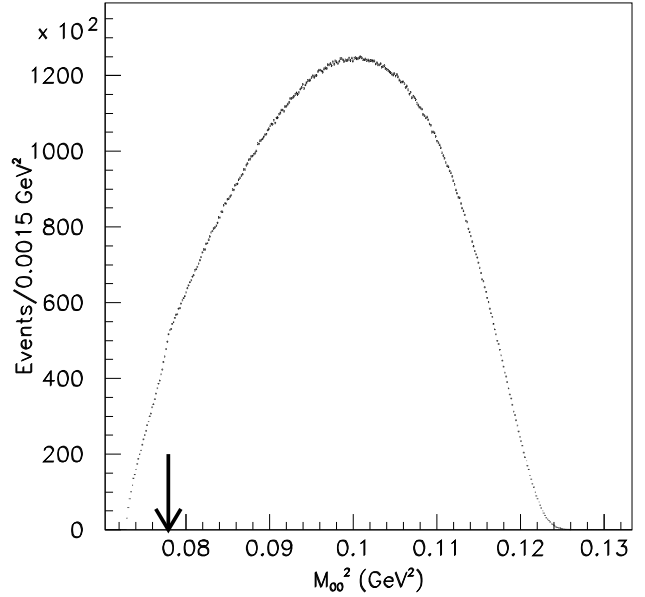

Figure 2. $\quad M_{\pi^{0} \pi^{0}}^{2}$ mass distribution in $K^{ \pm} \rightarrow$ $\pi^{ \pm} \pi^{0} \pi^{0}$. The arrow indicated the cusp position at $2 m_{\pi \pi}$.

$\pi^{0} \pi^{0}$ invariant mass distribution $\left(M_{00}^{2}\right)$ in $K^{ \pm} \rightarrow$ $\pi^{ \pm} \pi^{0} \pi^{0}$ decay. As shown in fig. 2 , at $2 m_{\pi}$ a singularity (cusp) appears; this cannot be explained by the simple parametrization of the matrix element [10]. The interpretation of this structure was given by [11] as due to the $\pi^{+} \pi^{-} \rightarrow \pi^{0} \pi^{0}$ strong rescattering, having different real and imaginary behaviour below and above the $2 \pi^{+}$production threshold. The fit of the $M_{00}^{2}$ shape distribution, taking into account the detector acceptance and including the description of one and two loops strong rescattering processes (as described in [12] (CI)), allows to extract $a_{0}-a_{2}$ and $a_{2}$. The selection of $\sim 60 \cdot 10^{6} K^{ \pm} \rightarrow \pi^{ \pm} \pi^{0} \pi^{0}$ is based on the LKr. Thanks to the sizable statistics collected and the good resolution on the $\pi^{0}$ mass given by the LKr, the cusp structure is very evident. The fit is performed excluding a small region around the threshold where the theoretical treatment of the electromagnetic interaction is still missing. The preliminary results obtained are:

$$
\begin{gathered}
\left(a_{0}-a_{2}\right) m_{\pi}=0.266 \pm 0.005_{\text {stat }} \pm 0.002_{\text {syst }} \\
\quad \pm 0.001_{\text {ext }} \pm 0.013_{\text {th }} \\
a_{2} m_{\pi}=-0.039 \pm 0.009_{\text {stat }} \pm 0.006_{\text {syst }} \pm 0.002_{\text {ext }}
\end{gathered}
$$

and using the ChPT constraint, between $a_{0}$ and $a_{2}$, mentioned above ( 1 parameter fit):

$$
\begin{aligned}
\left(a_{0}-a_{2}\right) m_{\pi}= & 0.268 \pm 0.003_{\text {stat }} \pm 0.002_{\text {syst }} \\
& \pm 0.001_{\text {ext }} \pm 0.013_{\text {th }}
\end{aligned}
$$

where the theoretical error is assigned to take into account the missing orders and Coulomb corrections. Recent results both in radiative corrections [13] and in the next order preliminary calculations [14] allows to decrease this contribution to the total error at level of $1 \%$ or $2 \%$. These results are in agreement with our previous results based on a partial set of data [15]. In a different approach given in [16] (CGKR) based on an effective non-relativistic Lagrangian, the electromagnetic effects are naturally included. The different structure of the expansion gives a different correlation between the parameters with respect to the CI approach. The preliminary results obtained using this alternative way are compatibles with the former:

$$
\begin{aligned}
& \left(a_{0}-a_{2}\right) m_{\pi}=0.273 \pm 0.005_{\text {stat }} \pm 0.002_{\text {syst }} \pm 0.001_{\text {ext }} \\
& a_{2} m_{\pi}=-0.065 \pm 0.015_{\text {stat }} \pm 0.010_{\text {syst }} \pm 0.002_{\text {ext }}
\end{aligned}
$$

and with the 1 parameter fit:

$\left(a_{0}-a_{2}\right) m_{\pi}=0.266 \pm 0.003_{\text {stat }} \pm 0.002_{\text {syst }} \pm 0.001_{\text {ext }}$

\section{CUSP IN $K_{L} \rightarrow \pi^{0} \pi^{0} \pi^{0}$}

Similar to $K^{ \pm} \rightarrow \pi^{ \pm} \pi^{0} \pi^{0}$, in the $K_{L} \rightarrow 3 \pi^{0}$ decay the cusp effect have to be visible thanks to the contribution of the rescattering in $K_{L} \rightarrow \pi^{+} \pi^{-} \pi^{0}$ [17]. Anyway, for dinamical reason, the effect is smaller and huge statistics is needed in order to extract results with error similar to the charged K. Na48 2000 data shows a discontinuity in $M_{\pi^{0} \pi^{0}}$ spectrum from $\sim 100 \cdot 10^{6} K_{L} \rightarrow 3 \pi^{0}$ decays (already presented, for instance, in [18]). Recently the KTEV collaboration [19] published a first measurement based on $\sim 68 \cdot 10^{6}$ events with a result, on $\left(a_{0}-a_{2}\right)$, marginally in agreement with the results in charged kaons, the ChPT prediction and the DIRAC result [20].

\section{COMPARISON AND CONCLUSIONS}

Two different ways to measure the $\pi \pi$ scattering lengths are exploited by NA48/2 in kaon 


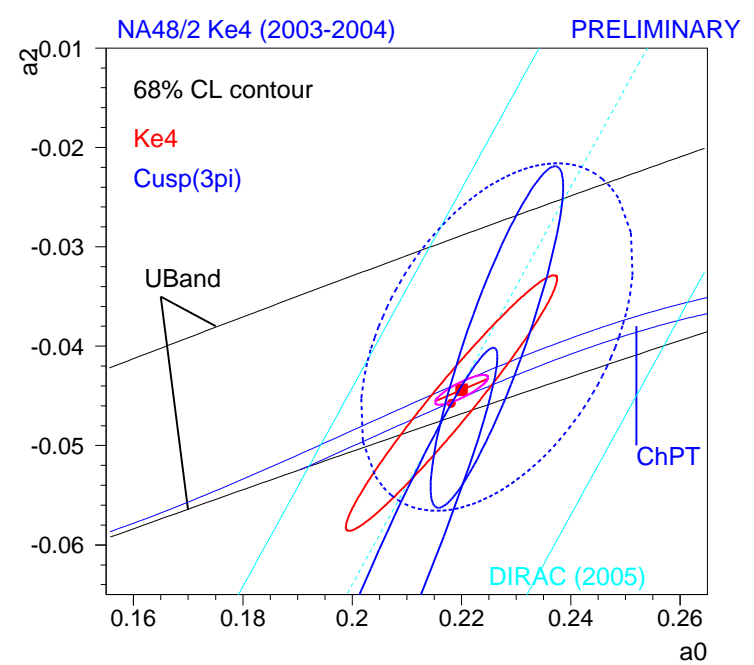

Figure 3. Final results

decays. The first is based on the measurements of the phase shift and the form factors in $K_{e 4}$ decay, the second on the accurate study of the Dalitz Plot distribution in $K^{ \pm} \rightarrow \pi^{ \pm} \pi^{0} \pi^{0}$ decay. In fig. 3 the agreement between the two independent measurement is shown together with other experimental results. The blue dotted ellipse represents the result with the CI theory assuming an additional $5 \%$ error while the inner ellipse is obtained with a more realistic $1 \%$ error. The other blue ellipse is the CGKR fit result. The $K_{e 4}$ measurement is shown with the red ellipse and the red point is the ChPT prediction [21].

\section{REFERENCES}

1. J. R. Batley et al. [NA48/2 Collaboration], Eur. Phys. J. C 52, 875 (2007) [arXiv:0707.0697 [hep-ex]].

2. V. Fanti et al. [NA48 Collaboration], Nucl. Instrum. Meth. A 574 (2007) 433.

3. N. Cabibbo and A. Maksymowicz, Phys. Rev. 137 (1965) B438.

4. G. Amoros, J. Bijnens and P. Talavera, Nucl.
Phys. B 585 (2000) 293 [Erratum-ibid. B 598 (2001) 665] [arXiv:hep-ph/0003258].

5. R. Brun, R. Hagelberg, M. Hansroul and J. C. Lassalle, CERN-DD-78-2-REV (http://wwwasd.web.cern.ch/wwwasd/geant/)

6. J. R. Batley et al. [NA48/2 Collaboration], Eur. Phys. J. C 54 (2008) 411.

7. S. M. Roy, Phys. Lett. B 36 (1971) 353.

8. J. Gasser, PoS KAON (2008) 033 [arXiv:0710.3048 [hep-ph]].

9. G. Colangelo, J. Gasser and H. Leutwyler, Nucl. Phys. B 603 (2001) 125 [arXiv:hep$\mathrm{ph} / 0103088]$.

10. W. M. Yao et al. [Particle Data Group], J. Phys. G 33 (2006) 1.

11. N. Cabibbo, Phys. Rev. Lett. 93 (2004) 121801 [arXiv:hep-ph/0405001].

12. N. Cabibbo and G. Isidori, JHEP 0503 (2005) 021 [arXiv:hep-ph/0502130]. Paper 1 to 1 of 1

13. M. Bissegger, A. Fuhrer, J. Gasser, B. Kubis and A. Rusetsky, arXiv:0807.0515 [hep-ph].

14. G. Isidori, private communication.

15. J. R. Batley et al. [NA48/2 Collaboration], Phys. Lett. B 633 (2006) 173 [arXiv:hepex/0511056].

16. G. Colangelo, J. Gasser, B. Kubis and A. Rusetsky, Phys. Lett. B 638 (2006) 187 [arXiv:hep-ph/0604084].

17. M. Bissegger, A. Fuhrer, J. Gasser, B. Kubis and A. Rusetsky, Phys. Lett. B 659 (2008) 576 [arXiv:0710.4456 [hep-ph]].

18. S. Giudici, talk given at QNP 2006, Madrid, 5-10 June 2006.

19. E. Abouzaid et al. $[\mathrm{KTeV}$ Collaboration], Phys. Rev. D $\mathbf{7 8}$ (2008) 032009 [arXiv:0806.3535 [hep-ex]].

20. B. Adeva et al. [DIRAC Collaboration], Phys. Lett. B 619 (2005) 50 [arXiv:hepex/0504044].

21. G. Colangelo, J. Gasser and H. Leutwyler, Phys. Rev. Lett. 86 (2001) 5008 [arXiv:hepph/0103063]. 